\title{
Erratum: Core Structure and Non-Abelian Reconnection of Defects in a Biaxial Nematic Spin-2 Bose-Einstein Condensate [Phys. Rev. Lett. 117, 275302 (2016)]
}

Magnus O. Borgh and Janne Ruostekoski (Received 1 March 2017; published 20 March 2017)

DOI: 10.1103/PhysRevLett.118.129901

The two separate conjugacy classes $\left\{\left(n, \pm i \sigma_{x}\right),\left(n, \pm i \sigma_{y}\right)\right\}$ and $\left\{\left(n, \pm i \sigma_{z}\right)\right\}$ were mistakenly grouped as a single class (iii). All other results and all conclusions remain unaffected. 